\title{
Guest Editorial: Special issue on mobility analytics for spatio-temporal and social data
}

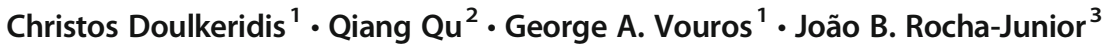

Published online: 9 July 2019

(C) Springer Science+Business Media, LLC, part of Springer Nature 2019

The aim of this special issue is to capture recent advances in developing data-intensive applications that analyze spatio-temporal/societal data, in order to foster the exchange of new ideas on multidisciplinary real-world problems, propose innovative solutions, and stimulate further research in the area of mobility data management and analysis.

Mobility analytics is a timely topic due to the ever-increasing number of diverse, real-life applications, ranging from social media to land, sea, and air surveillance systems, which produce massive amounts of streaming spatio-temporal data, whose acquisition, cleaning, representation, aggregation, processing and analysis pose new challenges for the data management community.

The papers published in this issue constitute revised and extended versions of the accepted papers of the 2nd International Workshop on Mobility Analytics for Spatio-Temporal and Social Data (MATES 2018), held in conjunction with the 44th International Conference on Very Large Databases (VLDB), in Rio de Janeiro, Brazil, on August 31, 2018.

MATES received 20 submissions, each of which was carefully reviewed by three different reviewers, in order to select 8 papers to be presented during the workshop in Rio. The authors of these papers were invited to submit an extended version to this special issue of Geoinformatica. After a second round of reviews, five papers have been accepted to be published in this special issue.

Christos Doulkeridis

cdoulk@unipi.gr

Qiang Qu

qiang@siat.ac.cn

George A. Vouros

georgev@unipi.gr

João B. Rocha-Junior

joao@uefs.br

1 University of Piraeus, Piraeus, Greece

2 Shenzhen Institutes of Advanced Technology, Chinese Academy of Sciences, Shenzhen, China

3 Universidade Estadual de Feira de Santana, Feira de Santana, Brazil 
The following papers appear in this special issue:

[1] "Regularized Topic-aware Latent Influence Propagation in Dynamic Relational Networks", by Shuhui Wang, Liang Li, Chenxue Yang and Qingming Huang;

[2] "On the Composition and Recommendation of Multi-Feature Paths: A Comprehensive Approach", by Vincenzo Cutrona, Federico Bianchi, Michele Ciavotta and Andrea Maurino;

[3] "A Hybrid CNN-LSTM Model for Typhoon Formation Forecasting”, by Rui Chen, Xiang Wang, Weimin Zhang, Xiaoyu Zhu, Aiping Li and Chao Yang;

[4] "A Spatially-Pruned Vertex Expansion Operator in the Neo4j Graph Database System", by Yuhan Sun and Mohamed Sarwat;

[5] "Spatio-temporal Mining of Keywords for Social Media Cross-social Crawling of Emergency Events", by Andrea Autelitano, Barbara Pernici and Gabriele Scalia;

These papers cover a range of important topics related to mobility analytics for spatio-temporal and social data. Wang et al. [1] investigate influence propagation in social networks and propose a framework for modeling topic-aware influence propagation in dynamic network structures. Cutrona et al. [2] present a novel approach to enrich trajectory representations, based on semantic annotations, and use this information to recommend trajectories based on the user preferences. Chen et al. [3] propose a new model to predict the formation and intensity of typhoons, using spatio-temporal correlation of atmospheric and ocean variables. Sun and Sarwat [4] develop a new query operator for graph database systems that store spatial data, which optimizes the execution of graph queries that involve spatial predicates. Autelitano et al. [5] propose a new method for extracting relevant images and videos from social media during an emergency event, dynamically mining event-related keywords, aiming at following the evolution of an event.

Collectively, these five papers cover a broad range of social, spatial and temporal data related problems. They present a large variety of challenges that are currently being investigated, where social, spatial and temporal data are being used to extract relevant information for the society. Moreover, they contain novel techniques and approaches that are deemed relevant for many emerging applications related to mobility analytics.

We would like to thank the authors for the excellent contributions to this issue; the reviewers for providing thoughtful and detailed manuscript reviews; as well as the editorial office staff at Springer for their assistance during the review process.

Publisher's note Springer Nature remains neutral with regard to jurisdictional claims in published maps and institutional affiliations. 


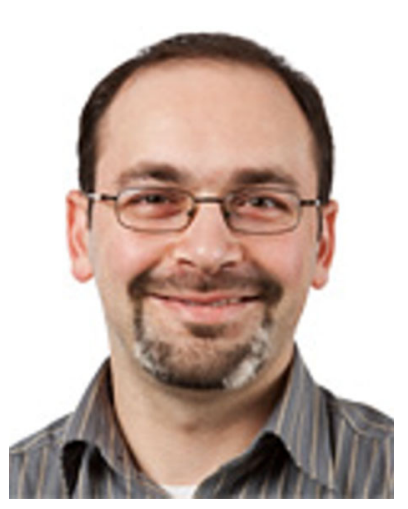

Christos Doulkeridis is an Assistant Professor at the Department of Digital Systems in the University of Piraeus. He has been awarded both a Marie-Curie and an ERCIM Allain Bensoussan fellowship for post-doctoral studies at the Norwegian University of Science and Technology in 2011 and 2009 respectively. Prior to this, he received his $\mathrm{PhD}$ from the Department of Informatics in the Athens University of Economics and Business in 2007. He has published in top international journals and conferences in the areas of data management, knowledge discovery and distributed systems. He was the winner of the competition in SemEval'17 Task 4: Sentiment Analysis on Twitter. His research interests include Big Data, cloud-based data management, distributed query processing, mobility analytics, spatial and spatio-temporal databases.

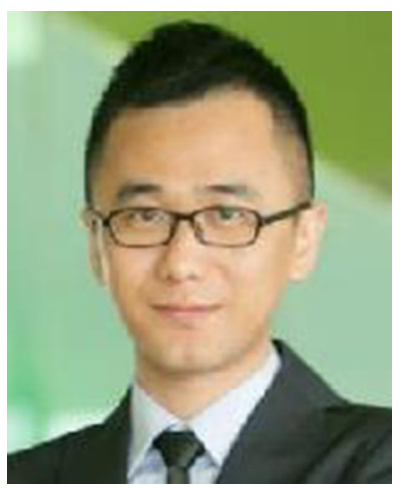

Qiang Qu is a professor at Shenzhen Institutes of Advanced Technology (SIAT), and University of Chinese Academy of Sciences, and the deputy director of Hangzhou Institute of Advanced Technology. Before joining SIAT, he worked at Carnegie Mellon University, ETH Zurich, Innopolis University, Nanyang Technological University and Singapore Management University. He is also an adjunct professor of Chinese University of HongKong (Shenzhen). His current research interests are in data-intensive applications and systems, focusing on efficient and scalable algorithm design, blockchain, data sense-making, and mobility intelligence. 


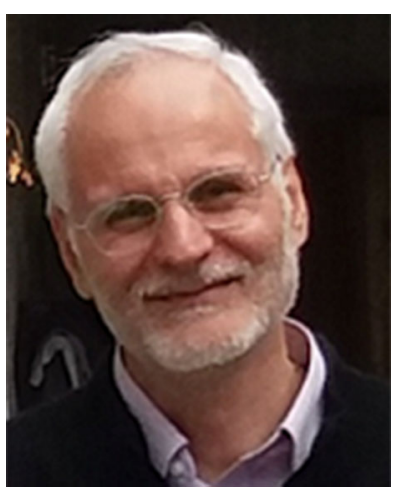

George A. Vouros (B.Sc, Ph.D) holds a BSc in Mathematics (1986), and a PhD in Artificial Intelligence (1992) all from the University of Athens, Greece. Currently he is a Professor in the Department of Digital Systems in the University of Piraeus and head of the AI-Lab in this Department. He has done research in the areas of Expert Systems, Knowledge Management, Collaborative Systems, Ontologies, and Agents and Multi-Agent Systems. He served/serves as program chair, chair and member of organizing / program committees of national and international conferences (ISWC, AAMAS, AAAI, IJCAI, ECAI, WI/IAT, AT, EUMAS, ICMLA, ESWC, CSCL, AIAI) and as member of steering committees/boards of international conferences/workshops (EURAMAS, SETN, AT, COIN). He is/was senior researcher in numerous EUfunded and National research projects (GSRT/AMINESS, FP7/Grid4A1l, FP7/SEMAGROW, COST/ Agreement Technologies the most recent ones), and he recently coordinated the successful DART project (SESAR project) and datACRON Big Data project (H2020 ICT-16). George Vouros has served for many years as chair of the Hellenic A.I. Society board. Further details concerning his work can be found in http://ai-group.ds.unipi.gr/georgev/.

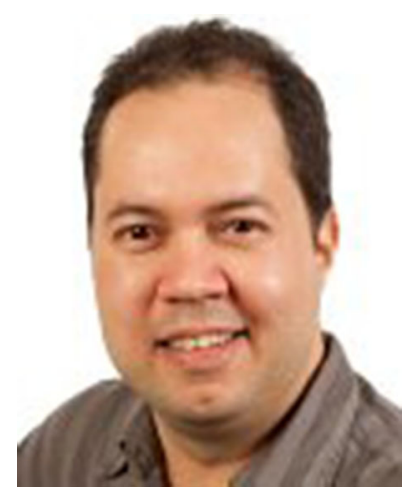

João B. Rocha-Junior is Professor at the Department of Exact Sciences at the State University of Feira de Santana (UEFS). He received his M.Sc. in Computer Science from the Federal University of Pernambuco (UFPE) in 2002. The M.Sc. was about the Search Architecture of Radix (a famous Brazilian search engine at that time), integrating data from different sources such as web pages, news and images. In 2012, he received the Ph.D. in Computer and Information Science from the Norwegian University of Science and Technology (NTNU). The $\mathrm{Ph} . \mathrm{D}$. thesis was about the Efficient Processing of Preference queries in Spatial and Distributed Databases. Today, Rocha-Junior is Professor in the Undergraduate Course of Computer Engineering at UEFS (EComp) and 
permanent member of the Graduate Program in Computer Science (PGCC). He has been Program Chair of some conferences on Databases such as Brazilian Symposium on Databases (SBBD) and International Asia-Pacific Web Conference, and reviewer of important journals such as The VLDB Journal, Journal of Computer Science and Technology (JCST), IEEE Transactions on Knowledge and Data Engineering (TKDE), International Journal of Communication Systems, IEEE Transactions on Services Computing (TSC), World Wide Web Journal (WWWJ) and GeoInformatica. He has publications in important conferences such as VLDB, EDBT and SSTD. His research interests include Spatial Keyword Query Processing, Efficient Processing of Preference Queries, Distributed Query Processing, Spatial Databases, and Information Retrieval. 